\title{
ANALYTICAL AND NUMERICAL STUDIES OF THE BONHOEFFER VAN DER POL SYSTEM
}

\author{
BELINDA BARNES and ROGER GRIMSHAW ${ }^{1}$
}

(Received 9 December 1994; revised 7 May 1995)

\begin{abstract}
The Bonhoeffer Van der Pol system is a planar autonomous nonlinear system of differential equations which has been invoked as a qualitative model of physiological states in a nerve membrane. It contains three independent parameters and previous work has only studied a small portion of the parameter space, that part which is thought to be of physiological relevance. Here we give a complete study of the full parameter space, using both theoretical results and numerical solutions.
\end{abstract}

\section{Introduction}

The Bonhoeffer Van der Pol (BVP) model is given by

$$
\begin{aligned}
& \dot{x}=c\left(x+Y-\frac{1}{3} x^{3}+z\right), \\
& \dot{Y}=\frac{1}{c}(-x-b Y+A),
\end{aligned}
$$

where $x, Y$ are the dependent variables, $\dot{x}, \dot{Y}$ denote derivatives with respect to the time $t$, and $A, b, c$ and $z$ are constant parameters, where $c>0$. Here $A, b$ and $c$ are control parameters, while $z$ represents an applied impulse. Since it is assumed to be a constant here, it can be removed by the following transformation

$$
y=Y+z, \quad a=A+b z,
$$

which transforms $(1.1 \mathrm{a}, \mathrm{b})$ into

$$
\begin{aligned}
& \dot{x}=c\left(x+y-\frac{1}{3} x^{3}\right), \\
& \dot{y}=\frac{1}{c}(-x-b y+a),
\end{aligned}
$$

${ }^{1}$ Department of Mathematics, Monash University, Clayton, Victoria 3168 Australia.

(C) Australian Mathematical Society, 1997, Serial-fee code 0334-2700/97 
The BVP system $(1.3 \mathrm{a}, \mathrm{b})$ is one of a class of nonlinear relaxation oscillators. For $a=b=0,(1.3 \mathrm{a}, \mathrm{b})$ reduces to the well-known Van der Pol equation, which has a unique stable periodic solution. The BVP system was introduced by Fitzhugh $[1,2]$ as a two-dimensional representation of the four-dimensional Hodgken-Huxley system. This latter system describes the transmission of neural impulses in the axon of a nerve, and was based on experimental data using the neuron of a squid giant axon. Fitzhugh [2] divided the Hodgkin-Huxley system into a two-dimensional excitory subsystem which changes rapidly, and a two-dimensional refractory subsystem which changes slowly. Then, using a linear projection, he eliminated a dimension from each subsystem to obtain the BVP system. While this reduction is essentially qualitative, Fitzhugh [2] claims that the BVP system retains the essential features governing behaviour close to critical values, and thus "provides a simplified but central unifying concept for the theoretical study of axon physiology". Subsequently the BVP system has been regarded as a useful qualitative model of a physiological system (for example, Rinzel [8]).

Fitzhugh [2] carried out some theoretical and numerical analyses of the BVP system for particular parameter values related to a physiological application (for example, $A=0.7, b=0.8, c=3$ and $z=-0.34$, so that $a=0.43$ ). The parameter values were chosen to ensure that the phase plane contained just a single critical point (either stable, or unstable) and there was the possibility of the existence of a unique stable periodic solution. Recently, Kloeden et al. [7] discussed the effect of low intensity noise on the BVP system, and found that for these parameter values, the periodic solution in the deterministic system is replaced by a noisy periodic solution in the stochastic system. In this paper it is our intention to provide a complete and consistent picture of the solution structure of the BVP system (1.3a, b), over a wide range of parameter values. Although we will not explicitly explore the physiological implications of our results, we contend that by examining the full parameter space we can establish the robustness of the BVP system as a model of physiological phenomena, bearing in mind that the parameters $a, b, c$ in $(1.3 \mathrm{a}, \mathrm{b})$ are at best only qualitatively related to actual physiological variables. In Section 2 we analyse the critical point structure of (1.3a, b), and in Section 3 we describe some theoretical results concerning periodic solutions. Then in Section 4 we describe our numerical results.

\section{Equilibrium states}

The critical points, or equilibrium states, are obtained by setting the right-hand side of $(1.3 \mathrm{a}, \mathrm{b})$ to zero (or equivalently by setting the right-hand side of $(1.1 \mathrm{a}, \mathrm{b})$ to zero). 


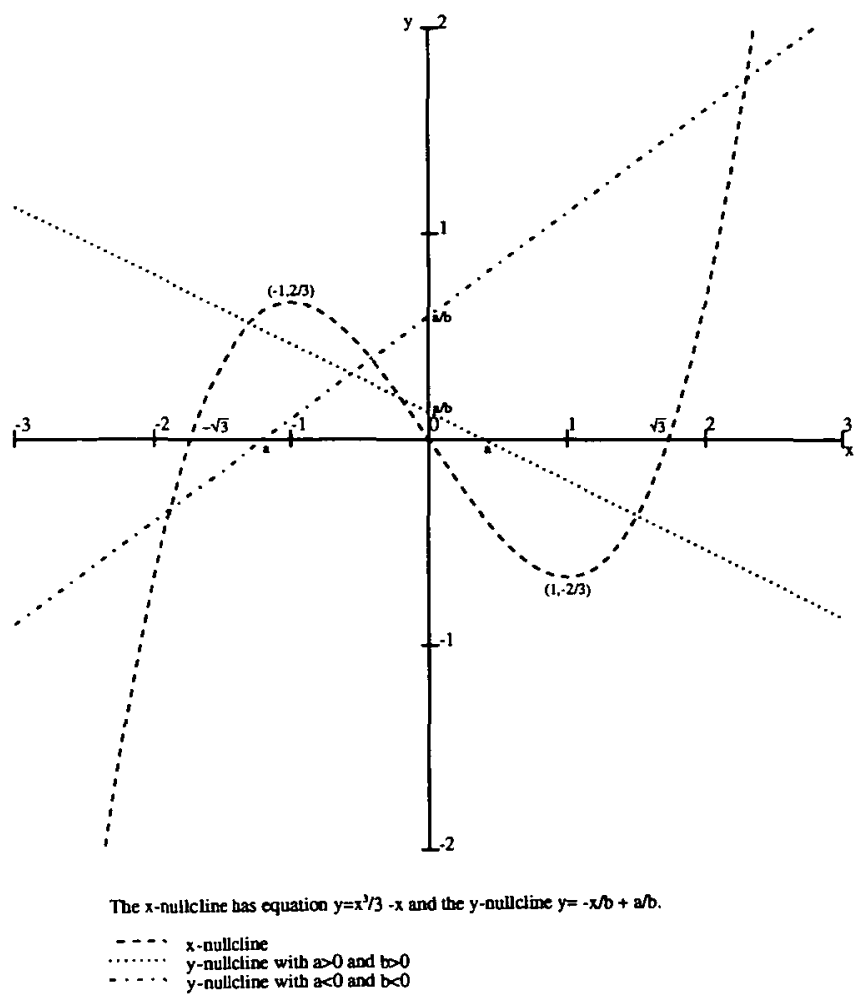

FIGURE 1. Phase plane for the BVP system

Hence they are given by $x=x_{0}, y=y_{0}$ where

$$
\begin{aligned}
y_{0} & =\frac{1}{3} x_{0}^{3}-x_{0}, \\
b y_{0} & =a-x_{0} .
\end{aligned}
$$

The $x$-nullcline is the cubic curve (2.1a) and is independent of the system parameters, while the $y$-nullcline is the straight line (2.1b) which depends on the parameters $a$ and $b$. Depending on these parameters there are either one, or three, intersections which determine the critical points. The situation is sketched in Figure 1. Alternatively, eliminating $y_{0}$ between $(2.1 \mathrm{a}, \mathrm{b})$ we obtain the cubic equation

$$
x_{0}^{3}+\frac{3(1-b)}{b} x_{0}-\frac{3 a}{b}=0 \text {, }
$$

which has either one, or three, real solutions for $x_{0}$. We find that there are three critical points if

$$
\frac{1-b}{b}<0 \quad \text { and } \quad\left|\frac{3 a}{b}\right|<2\left|\frac{1-b}{b}\right|^{3 / 2} \text {, }
$$



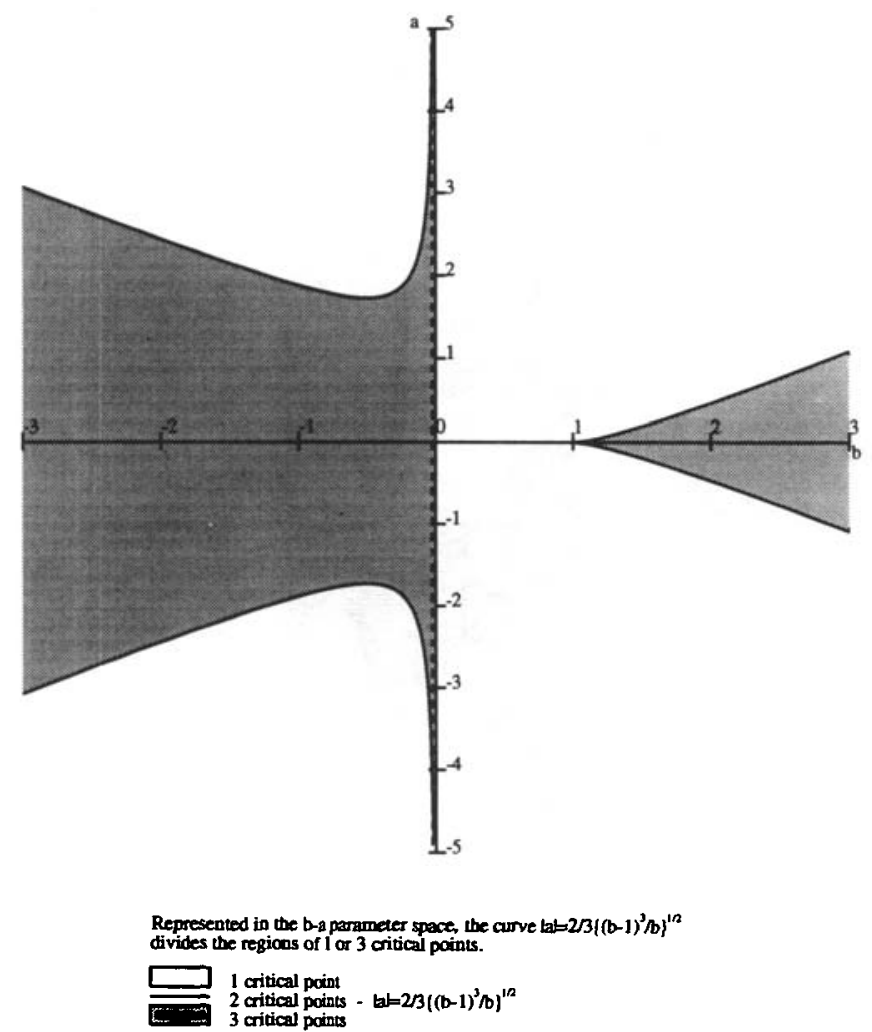

FIGURE 2. Number of critical points for the BVP system

and just one critical point otherwise. Exceptional cases occur when $b=0$ or $b=1$, both of which yield just one critical point, or when $|3 a / b|=2|(1-b) / b|^{3 / 2}$ which gives three critical points, two of which are coincident. The classification of the critical points with respect to the parameters $a, b$ is sketched in Figure 2. Next we note that the cubic equation (2.2) can be solved explicitly as follows.

$$
\begin{gathered}
b=0 ; \quad x_{0}=a, \\
b=1 ; \quad x_{0}=|3 a|^{1 / 3} \operatorname{sign} a, \\
a=0 \quad \text { and } \quad \frac{1-b}{b}>0 ; \quad x_{0}=0, \\
a=0 \text { and } \frac{1-b}{b}<0 ; \quad x_{0}=0 \quad \text { and } \quad x_{0}= \pm\left\{3\left|\frac{1-b}{b}\right|^{1 / 2},\right. \\
\frac{1-b}{b}>0 ; \quad x_{0}=2\left(\frac{1-b}{b}\right)^{1 / 2} \sinh \theta
\end{gathered}
$$


where

$$
\begin{gathered}
\sinh 3 \theta=\frac{3 a}{2 b}\left(\frac{1-b}{b}\right)^{-3 / 2} \\
\frac{1-b}{b}<0 \text { and }\left|\frac{3 a}{b}\right|>2\left|\frac{1-b}{b}\right|^{3 / 2} ; \quad x_{0}=2 \operatorname{sign}\left(\frac{a}{b}\right)\left(\frac{b-1}{b}\right)^{1 / 2} \cosh \theta
\end{gathered}
$$

where

$$
\begin{gathered}
\cosh 3 \theta=\left|\frac{3 a}{2 b}\right|\left(\frac{b-1}{b}\right)^{-3 / 2} \\
\frac{1-b}{b}<0 \text { and }\left|\frac{3 a}{b}\right|<2\left|\frac{1-b}{b}\right|^{3 / 2} ; \quad \text { let } \cos 3 \theta=\frac{3 a}{2 b}\left(\frac{b-1}{b}\right)^{-3 / 2}
\end{gathered}
$$

and

$$
x_{0}=2\left(\frac{b-1}{b}\right)^{1 / 2} \cos \theta,\left(\frac{b-1}{b}\right)^{1 / 2}\{-\cos \theta \pm \sqrt{3} \sin \theta\} .
$$

Note in particular that if $a=0$ then there is always one critical point at the origin, and if there are two other critical points then they are symmetrically placed with respect to the origin.

The stability of these critical points is determined by the eigenvalues of the matrix obtained by linearization of the right-hand side of $(1.3 \mathrm{a}, \mathrm{b})$ about $\left(x_{0}, y_{0}\right)$, given by

$$
\left[\begin{array}{cc}
c\left(1-x_{0}^{2}\right) & c \\
-1 / c & -b / c
\end{array}\right]
$$

Denoting these eigenvalues by $\lambda$ they are solutions of the quadratic equation

$$
\lambda^{2}-\lambda\left\{c\left(1-x_{0}^{2}\right)-b / c\right\}+1-b\left(1-x_{0}^{2}\right)=0 .
$$

Here $x_{0}$ is given by one of the expressions in $(2.4 \mathrm{a}-\mathrm{g})$, and there is, in general, a very complicated dependence of the eigenvalues $\lambda$ on the system parameters $a, b, c$. Here we are particularly interested in whether the critical point is a saddle point, stable or unstable node, or stable or unstable focus since each of these is structurally stable with respect to the present linearization and hence this classification can assist with the construction of phase portraits for the system $(1.3 \mathrm{a}, \mathrm{b})$. When the critical point is a centre it corresponds to a periodic solution in the linearized system but whether or not this periodic solution can exist in the full system requires examination of the nonlinear terms. The question as to whether periodic solutions can occur is taken up in Section 3. The classification of the critical points is most easily done from (2.6) 
using $b$ and $x_{0}$ as parameters for fixed values of $c$. We find that for each critical point $x_{0}$ the classification is,

Saddle point: $\quad b\left(1-x_{0}^{2}\right)>1$

Stable node: $\quad b\left(1-x_{0}^{2}\right)<1, c^{2}\left(1-x_{0}^{2}\right)<b$, and $\left\{c\left(1-x_{0}^{2}\right)+b / c\right\}^{2}>4 ;(2.7 \mathrm{~b})$

Unstable node: $b\left(1-x_{0}^{2}\right)<1, c^{2}\left(1-x_{0}^{2}\right)>b$, and $\left\{c\left(1-x_{0}^{2}\right)+b / c\right\}^{2}>4 \quad(2.7 \mathrm{c})$

Stable focus: $\quad b\left(1-x_{0}^{2}\right)<1, c^{2}\left(1-x_{0}^{2}\right)<b$, and $\left\{c\left(1-x_{0}^{2}\right)+b / c\right\}^{2}<4 ;(2.7 \mathrm{~d})$

Unstable focus: $b\left(1-x_{0}^{2}\right)<1, c^{2}\left(1-x_{0}^{2}\right)>b$, and $\left\{c\left(1-x_{0}^{2}\right)+b / c\right\}^{2}<4 ;(2.7 \mathrm{e})$

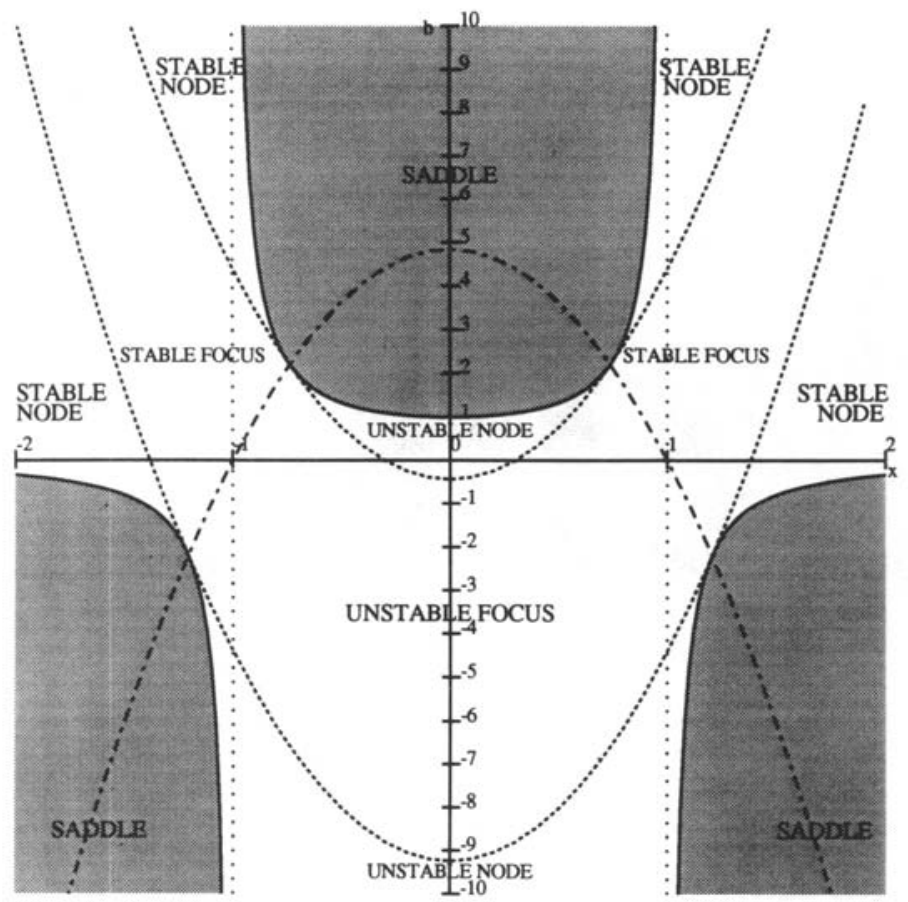

Represented in the $x-b$ phase plane for the case of $O 1$

$-b=1 /\left(1-x^{2}\right)$

(a) $\quad \mathrm{c}+\mathrm{c}\left(1-\mathrm{x}^{2}\right)=2$

FIGURE 3(I) Critical point classification for the BVP system

The situation is sketched in terms of the parameters $b, x_{0}$ in Figure 3. Note that in general the transition from stable to unstable occurs either when $b\left(1-x_{0}^{2}\right)=1$ and $c^{2}\left(1-x_{0}^{2}\right)<b$, corresponding to the transition from a stable node to a saddle 


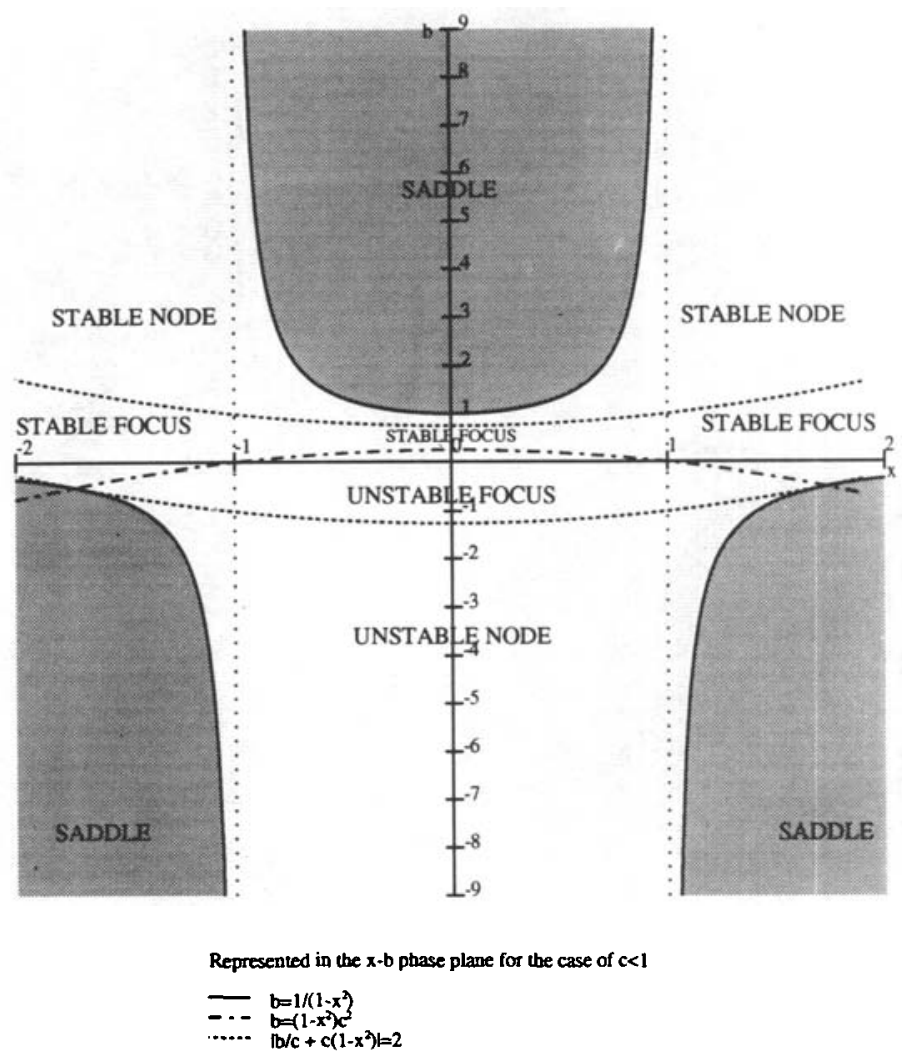

FIGURE 3(II) Critical point classification for the BVP system

point, or when $c^{2}\left(1-x_{0}^{2}\right)=b$ and $b\left(1-x_{0}^{2}\right)<1$, corresponding to a centre and the transition from a stable focus to an unstable focus. Both these transitions correspond to bifurcations, and we note in particular that the latter corresponds to an AndronovHopf bifurcation leading to the possible existence of a periodic solution; this aspect we shall examine further in Section 3. Also, we note that the curves $b\left(1-x_{0}^{2}\right)=1$ and $c^{2}\left(1-x_{0}^{2}\right)=b$ touch at the points $b=c$ for $c>1$ only, and at $b=-c$ for all $c>0$, while the curves $c\left(1-x_{0}^{2}\right)+b / c= \pm 2$ intersect both of these curves at the same points. This observation serves to distinguish the cases $0<c<1$ and $c>1$, and establishes the generic nature of each of these cases.

Because $x_{0}$ is itself a function of the parameters $a, b$ (see (2.4a-g)), the description of the stability properties of the critical points with respect to the parameters $a, b, c$ is quite complicated, and must be deduced by combining the information contained in Figures 2 and 3 (or alternately (2.4a-g) and (2.7a-e)). We choose here to fix the parameters $a$ and $c$, and vary $b$. The critical points are then defined in Figure 3(ii) 

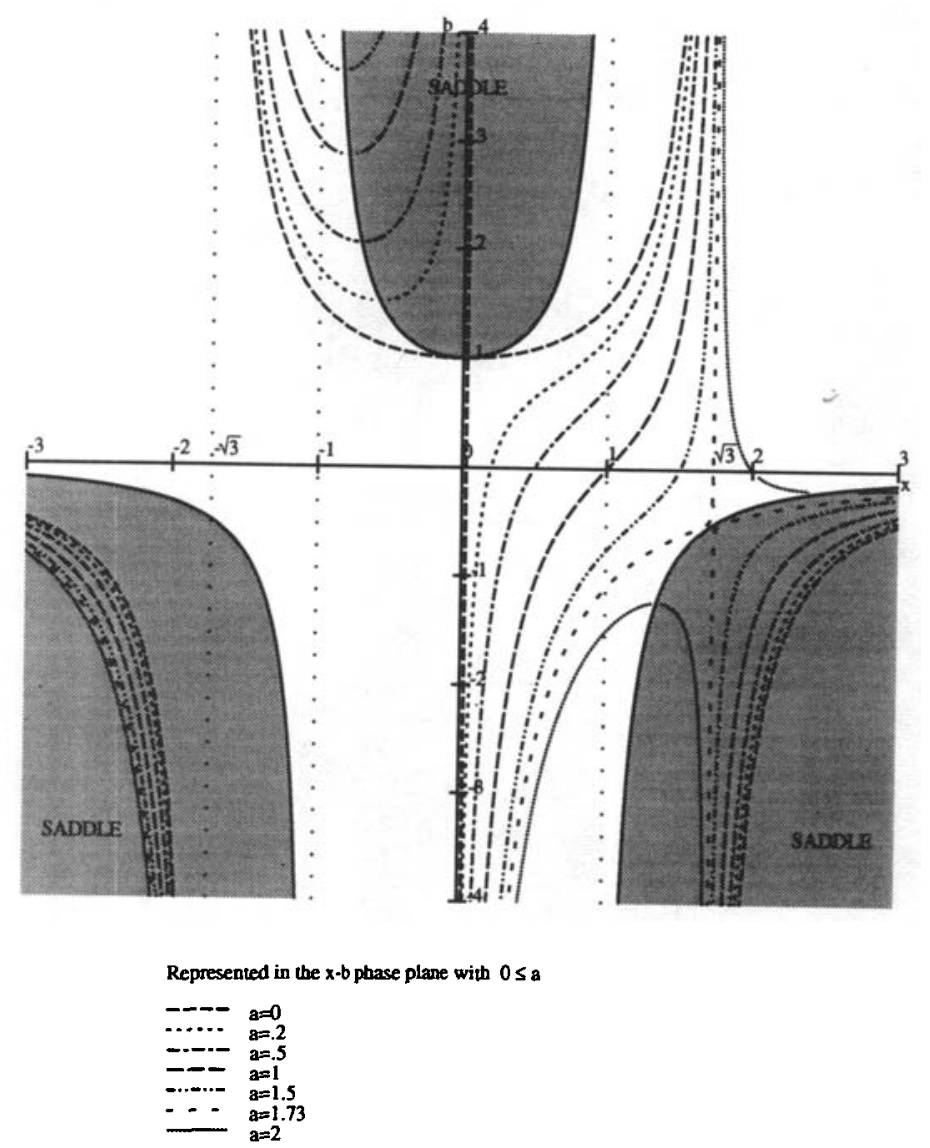

FIGURE 3(III) Critical point classification for the BVP system

which gives $b$ in terms of $x_{0}$ for different values of the parameter $a$. It is relevant to note that the turning points where $d b / d x_{0}=0$ on a critical point branch correspond to the condition $b\left(1-x_{0}^{2}\right)=1$, and hence correspond to the transition from a saddle point to a node. This is a saddle-node bifurcation, and as $b$ is varied this bifurcation describes the birth of a pair of critical points, a saddle point and a node. From Figure 3(ii) we see that this occurs for $b>0$ in the region $b>1$ as $b$ increases, and the emerging node is stable. For $b<0$, this can only occur when $|a|>\sqrt{3}$, and there are two such bifurcations; one occurs as $|b|$ decreases towards zero and the emerging node is stable; the other occurs as $|b|$ increases towards infinity, and the emerging node is stable (unstable) according as $b>-c(\leq-c)$. Apart from these saddle-node bifurcations, a change of stability can only occur on a critical point branch as $b$ is varied (see Figure 3(ii) and Figure 3) when the curve $c^{2}\left(1-x_{0}^{2}\right)=b$ is crossed (with 
$b\left(1-x_{0}^{2}\right)<1$ ) which corresponds to the transition from a stable focus to an unstable focus, and the possibility of an Andronov-Hopf bifurcation to a periodic solution. A more detailed description of the critical point parameter dependence and possible bifurcations follows.

(i) $a=0: \quad$ In this case the system $(1.3 \mathrm{a}, \mathrm{b})$ is symmetric with respect to the transformation $x, y \rightarrow-x,-y$. When $b=0$, the system $(1.3 \mathrm{a}, \mathrm{b})$ reduces to the Van der Pol equation (1.4) with a single unstable critical point at the origin, which is an unstable focus for $0<c<2$ and an unstable node for $c>2$. For $0<c \leq 1$, and as $b$ increases, this critical point changes from an unstable focus to a stable focus at $b=c^{2}$, and then changes to a stable node at $b=2 c-c^{2}$ before becoming an unstable saddle point at $b=1$. Further for $b \geq 1$ there is a pitchfork bifurcation and the two further critical points appear, symmetrically placed with respect to the origin. These are both stable nodes and as $b \rightarrow \infty$, the critical points become $( \pm \sqrt{3}, 0)$. We note that if $0<c<\sqrt{3}-1$ they are stable nodes for all $b \geq 1$, but if $\sqrt{3}-1<c<1$ become stable foci for $\left|b-\left(c^{2}+c\right)\right|<c\left(c^{2}+2 c-2\right)^{1 / 2}$.

For $c>1$, and as $b$ increases from zero, the critical point at the origin remains unstable. For $1<c<2$ it is an unstable focus for $0<b<c(2-c)$, then becomes an unstable node for $c(2-c)<b<1$, before becoming a saddle point for $b>1$. For $c>2$ it is an unstable node for $0<b<1$, and a saddle point for $b>1$. For $b>1$ there is a pitchfork bifurcation with two further critical points appearing, symmetrically placed with respect to the origin. These are both unstable for $1<b<-c^{2}+c\left(c^{2}+3\right)^{1 / 2}$ and are then stable for $b>-c^{2}+c\left(c^{2}+3\right)^{1 / 2}$. They are foci for $\left|b-\left(c^{2}+c\right)\right|<c\left(c^{2}+2 c-2\right)^{1 / 2}$ and nodes otherwise, and as $b \rightarrow \infty$ approach the points $( \pm \sqrt{3}, 0)$. Further, as shown above, the transition from unstable to stable occurs at a value of $b$ for which these critical points are foci, and hence can lead to the possible existence of a periodic solution through an Andronov-Hopf bifurcation.

For $b<0$ there are three critical points. One is at the origin, and is always unstable; it is a focus if $-c(c+2)<b<\min (0, c(2-c))$ and a node otherwise. The other two critical points are symmetrically placed with respect to the origin, and are saddle points.

(ii) $0<|a|<1$ : When $a \neq 0$ we cannot obtain the same detailed quantitative information as in (i) above. However the general pattern can be deduced from Figures 2 and 3. When $b=0$ there is a single critical point, which is unstable and a focus for $c\left(1-a^{2}\right)<2$ and a node otherwise. As $b$ increases, this critical point may change from a focus to a node, or vice-versa, and changes stability where $b=c^{2}\left(1-x_{0}^{2}\right)$. This transition is always from an unstable to a stable focus as shown above. As $b \rightarrow \infty$, this critical point approaches $(\sqrt{3}, 0)$ and is a stable node. Also, as $b$ increases two further critical points emerge through a saddle-node bifurcation for some $b>1$. 
These critical points have the same sign for $x_{0}$ and the opposite sign to the original critical point. One of these emerging critical points is a saddle point, which always lies between the other two critical points, and the other is initially a node, stable for $0<c \leq 1$, or $b>c>1$, and unstable for $c>b>1$. As $b$ continues to increase the saddle point approaches the origin, while the other critical point may change from a node to a focus, or vice-versa, is stable for $0<c \leq 1$, or $b>c>1$ and unstable for $c>b>1$, and approaches $(-\sqrt{3}, 0)$ as $b \rightarrow \infty$ where it is a stable node.

For $b<0$, there are three critical points. That closest to the origin is always unstable and is either a node or a focus; it is the continuation of the single critical point when $b=0$. The other two critical points are both saddle points, have $x_{0}>0(<0)$ respectively, and approach the points $( \pm \sqrt{3}, 0)$ as $b \rightarrow-\infty$.

(iii) $1 \leq|a|<\sqrt{3}$ : When $b=0$ there is a single critical point which is stable and a focus for $c\left(a^{2}-1\right)<2$ and a node otherwise. As $b$ increases this critical point remains stable and may change from a focus to a node, or vice-versa. Also as $b$ increases two further critical points emerge through a saddle-node bifurcation for some value of $b>1$. The properties of these critical points are similar to those in case (ii) above.

For $b<0$, there are three critical points. That closest to the origin is initially stable, and is either a node or a focus, being the continuation of the single critical point when $b=0$. It changes stability where $b=c^{2}\left(1-x_{0}^{2}\right)$ corresponding to the transition from a stable to an unstable focus. The other two critical points are both saddle points, have $x_{0}>0(<0)$ respectively, and approach the points $( \pm \sqrt{3}, 0)$ as $b \rightarrow-\infty$.

(iv) $|a| \geq \sqrt{3}$ : $\quad$ For $b \geq 0$ the situation is similar to that described in case (iii) above. For $b<0$ and sufficiently small, there is a stable critical point, either a focus or a node, lying between two saddle points with $x_{0}>0(<0)$ respectively. As $b$ decreases, the stable critical point and the saddle point of the same sign coalesce through a reverse saddle-node bifurcation, after which only a single critical point, the saddle point of the opposite sign can exist. If $c$ is sufficiently small, the stable critical point may change stability before coalescence; this occurs at $b=c^{2}\left(1-x_{0}^{2}\right)$ being the transition from a stable to an unstable focus. As $b$ decreases still further, eventually two more critical points emerge through a saddle-node bifurcation, of opposite sign to the persisting saddle point. One of these critical points is always a saddle point. If they emerge for $b \leq-c$, the other critical point is initially an unstable node, and remains unstable as $b$ decreases, being either a node or a focus. But if $b>-c$, the emerging critical point is initially a stable node, and as $b$ decreases may be either a node or focus, losing stability at $b=c^{2}\left(1-x_{0}^{2}\right)$, which corresponds to the transition from a stable to an unstable focus. As $b \rightarrow-\infty$ this critical point approaches the origin, and the saddle points approach $( \pm \sqrt{3}, 0)$. 


\section{Periodic solutions and phase portraits}

We now turn our attention to the question of whether periodic solutions can occur, and if so, whether they are stable or unstable. When $a=b=0$ in $(1.3 \mathrm{a}, \mathrm{b})$ the system reduces to the Van der Pol system which is known to have a unique stable periodic solution. In Figures 4 and 5 we show some typical phase portraits of the system (1.3a, b) when $b>0$ and $<0$ respectively, and also when $a=0$ and $c=3$. We see that in Figure 4(i) with $b=0.8$ there is a single critical point at the origin which is an unstable node, surrounded by a stable periodic solution; this situation is similar to that in the Van der Pol equation. In Figure 4(ii) with $b=1.28$ there are three critical points, a saddle point at the origin and unstable foci at $x_{0}= \pm-0.81, y_{0}=\mp 0.63$, surrounded by a stable periodic solution. In Figure 4(iii) with $b=2$ there are again three critical points, a saddle point at the origin and stable foci at $x_{0}= \pm-1.22, y_{0}=\mp 0.61$, and apparently no stable periodic solutions in this case. In Figure 5(i) with $b=-1$ there are three critical points, an unstable node at the origin and two saddle points at $x_{0}= \pm 2.45, y_{0}= \pm 2.45$, a stable periodic solution surrounds the origin but excludes the outlying unstable saddle points; a situation which has some similarity with the Van der Pol equation. In Figure 5(ii) with $b=1.5$ there are three critical points, an unstable node at the origin and two saddle points at $x_{0}= \pm 1, y_{0}= \pm 0.67$, and apparently no stable periodic solutions in this case.

To assist in interpreting these results, we next consider some general results to determine in what parameter regimes periodic solutions may or may not occur. Then we consider an existence theorem for periodic solutions similar to that used for Lienard equations (see, for instance, Grimshaw [3, p. 164]). This is followed by the use of Andronov-Hopf bifurcation theory to construct periodic solutions near parameter values where there is a transition from a stable to an unstable focus. Then in Section 4 we present some numerical results.

3.1. Preliminary results: First we consider Bendixson's criterion for the nonexistence of a periodic solution. This states that for a system

$$
\dot{x}=f(x, y), \quad \dot{y}=g(x, y)
$$

there can be no periodic solutions in any region where $\left(f_{x}+g_{y}\right)$ never vanishes (see, for instance, Grimshaw [3, p. 144] or Wiggins [10]). Applying this criterion to the system $(1.3 \mathrm{a}, \mathrm{b})$ we deduce that there are no periodic solutions where $\left\{c\left(1-x^{2}\right)-b / c\right\}$ never vanishes. This implies in particular that $b<c^{2}$ is a necessary condition for the existence of a periodic solution. Further, for any periodic solution there exists at least one value of $x$, say $\hat{x}$, such that $\hat{x}^{2}=\left(1-b / c^{2}\right)$.

Index theory can also be used to locate where periodic solutions may be found (see, for instance, Grimshaw [3, p. 145-153], or Wiggins [10]). Here we recall that the 


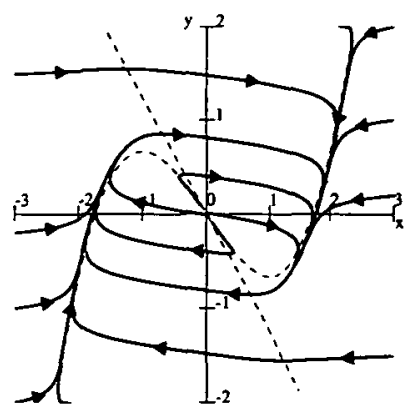

Parameters a b. c:- $\quad 0.0 \quad 0.8 \quad 3.0$

(i)

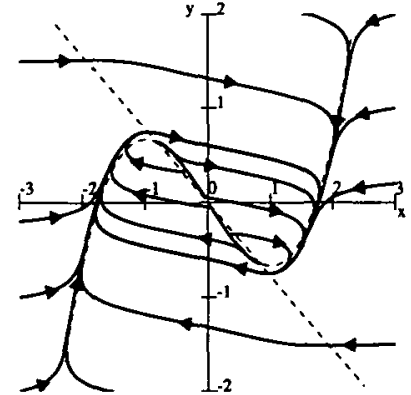

Paramecers a b. c:- $0.0 \quad 1.28 \quad 3.0$

(ii)

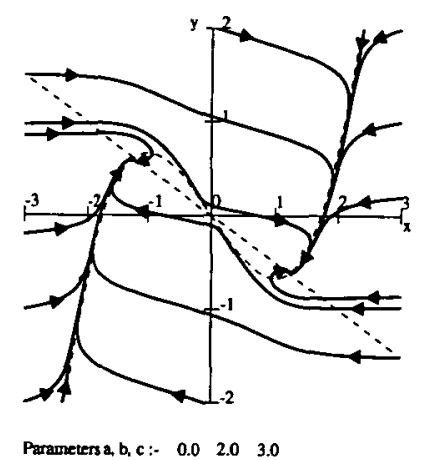

(iii)

FIGURE 4. Phase plane for the BVP system

index of a critical point is 1 if it is a focus, centre or node, and is -1 if it is a saddle point. Then any periodic solution corresponds to a closed orbit in the $x-y$ phase plane which must contain at least one critical point, and the sum of the indices of the critical points lying within the closed orbit is 1 . For the system $(1.3 \mathrm{a}, \mathrm{b})$, this implies that for $b \geq 0$, any periodic solution must contain a saddle point with two nodes or foci. For $b<0$, any periodic solution must enclose just a single node or focus.

Finally we recall from our analysis in Section 2 that periodic solutions may emerge from an Andronov-Hopf bifurcation where there is a transition from a stable to an unstable focus. From this analysis this occurs where $c^{2}\left(1-x_{0}^{2}\right)=b$ with $b\left(1-x_{0}^{2}\right)<1$ and can only occur where $-c<b<c$. The application of Andronov-Hopf bifurcation theory to this transition is described in 3.4 below.

3.2. Non-existence of periodic solutions: Based on the considerations in 3.1 above, the regions in parameter space where periodic solutions cannot occur are shown in 


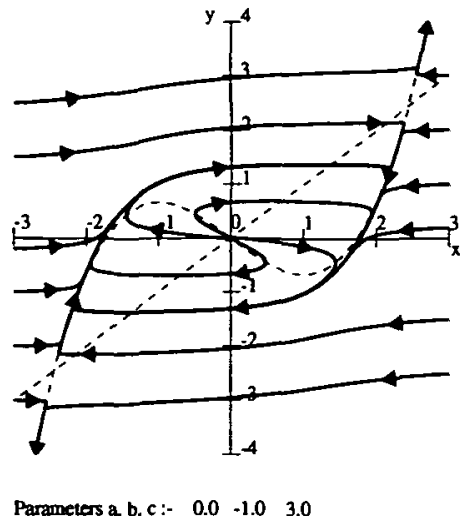

(i)

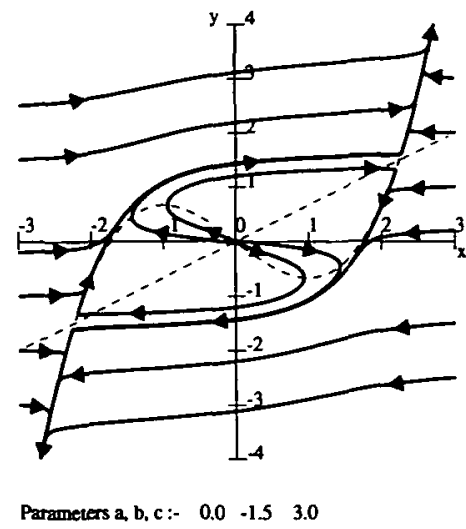

(ii)

FIGURE 5. Phase plane for the BVP system

Figures 6 and 7, for the $b-c$ and $b-a$ parameter spaces respectively, where either $a$ or $c$ is kept fixed. The dark-shaded regions indicate where periodic solutions cannot exist. In the $b-c$ parameter space the boundary $b=c^{2}$ is determined by the Bendixson criterion, and the boundary $2 b=-3 c^{2}-c\left(16+9 c^{2}\right)^{1 / 2}$ is determined from index theory, while in the $b-a$ parameter space the boundary $|3 a / b|=2\left|\left(\frac{1-b}{b}\right)\right|^{3 / 2}$ with $b<0$ is determined from index theory. Further information can be obtained by combining index theory with a consideration of the direction fields of $(1.3 \mathrm{a}, \mathrm{b})$. Thus for $b<0$, and for the case when there are three critical points (that is, (2.3) holds), the two outlying critical points are both saddle points (see Figure 3 ) and any periodic solution must contain the interior critical point and exclude the outlying saddle points, while also satisfying the Bendixson criterion. It is readily shown that this is impossible if the outlying saddle points lie in the region $|x|<2$ (see Figure 1). This leads to the criterion that there are no periodic solutions for $b\left(1-x_{0}^{2}\right)<0$ and $|a|<-2 b / 3-2$. Similarly it can be shown that for $b>0$ and again for the case when there are three critical points that there are no periodic solutions for $|a|<2 b / 3-2$.

Now it does not necessarily follow that periodic solutions do exist in the remaining regions of the parameter space. However, we will show in the following sub-sections that in a certain region near the origin in the $b-a$ parameter space, with $-c<b<$ $\min \left(c, c^{2}\right)$ periodic solutions do exist. Further progress in defining the regions where periodic solutons may occur can be obtained by assuming that where a periodic solution occurs it is unique, and confining attention to the existence of a stable periodic solution. In this case index theory shows that the periodic solution must enclose an unstable node or focus. If $x_{0}$ is this critical point then from (2.7) we see that $b\left(1-x_{0}^{2}\right)<1$ and 


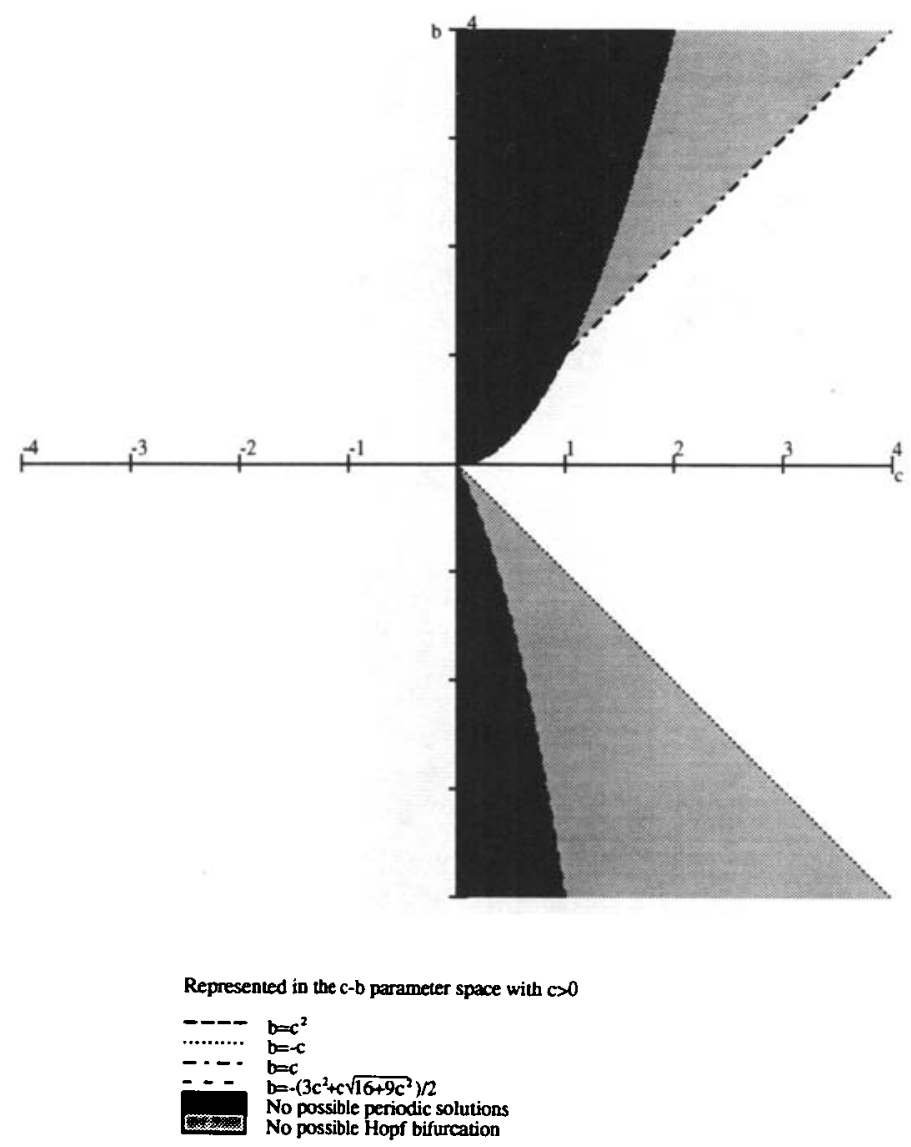

FIGURE 6. Areas where no periodic solutions can occur

$c^{2}\left(1-x_{0}^{2}\right)>b$. It follows that there can be no such unique stable periodic solution if $b>c$. Further, by combining the above criteria with (2.2) it can be shown that there is no unique stable periodic solution if $b>0$ and $|a|<2 b / 3-1$, in which case there are three critical points so that (2.3) is satisfied, while if $b>0$ and there is just a single critical point then there is no unique stable periodic solution if $|a|>-2 b / 3+1$. The lightly-shaded regions in Figures 6 and 7 indicate these zones where there can be no unique stable periodic solution. A further exclusion zone can be obtained by assuming that any periodic solution must emerge from an Andronov-Hopf bifurcation which requires $b^{2}<c^{2}$, and hence we can add the regions $b>c$ and $b<-c$ as excluding this possibility.

In summary Figures 6 and 7 indicate exclusion zones for periodic solutions, some of which are absolute (darkly-shaded) and the others (lightly-shaded) are conditional. 

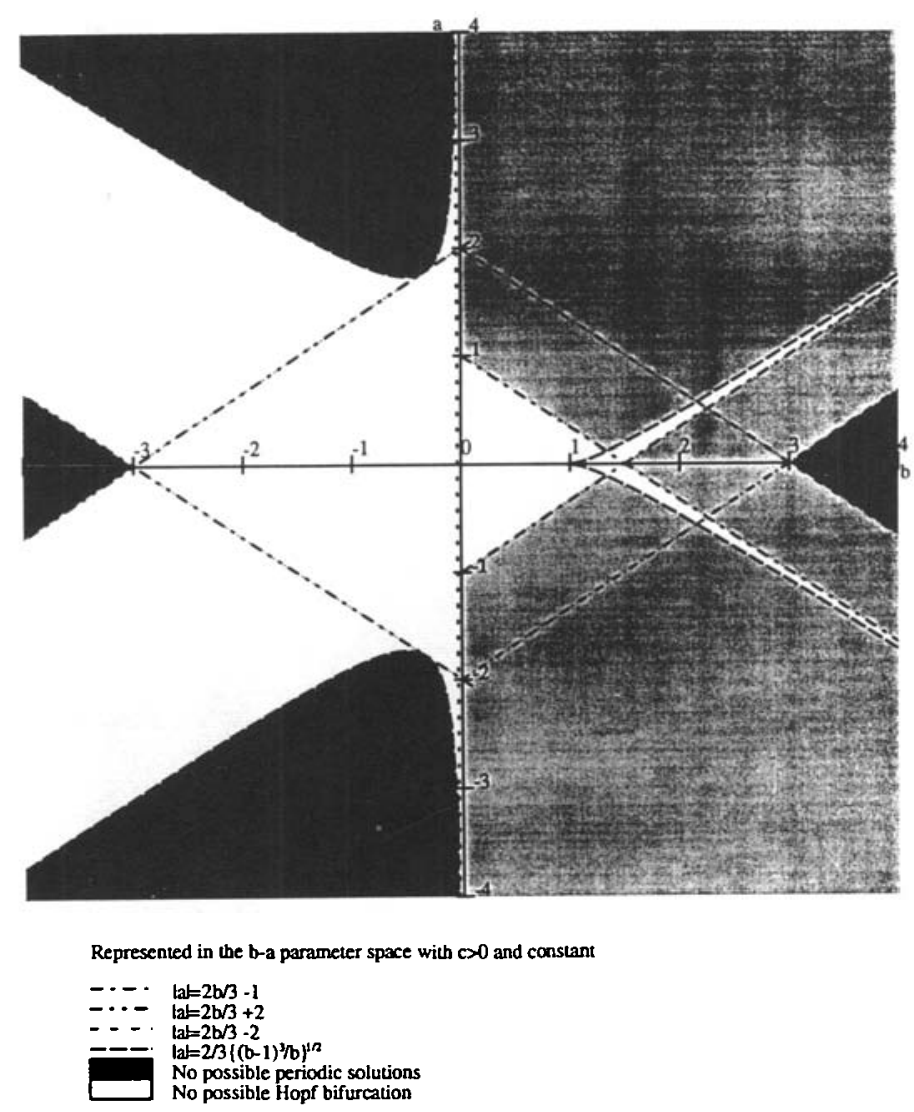

FIGURE 7. Areas where no periodic solution can occur

But let us note here that our numerical calculations did not find any periodic solutions in any exclusion zone, either absolute or conditional.

3.3. Existence of Periodic Solutions: Equations (1.3a, b) can be combined to give the single second-order differential equation,

$$
\ddot{x}+f(x) \dot{x}+g(x)=0,
$$

where

$$
f(x)=c\left(x^{2}-1\right)+\frac{b}{c}
$$

and

$$
g(x)=x(1-b)+\frac{1}{3} b x^{3}-a
$$


Equation (3.1a) is a Lienard equation and can also be written as a pair of first-order equations

$$
\begin{aligned}
\dot{x} & =Y-F(x), \\
\dot{Y} & =-g(x),
\end{aligned}
$$

where

$$
F(x)=\int_{0}^{x} f(u) d u .
$$

Note that $Y=c y+b x / c$, and so $(3.2 \mathrm{a}, \mathrm{b})$ is an alternative form to the original system $(1.3 \mathrm{a}, \mathrm{b})$. It is known that $(3.1 \mathrm{a})$, or $(3.2 \mathrm{a}, \mathrm{b})$ has a unique stable periodic solution if (see, for instance, Jordan and Smith, [6])

(i) $\quad F(x)=-F(x)$ for all $x$.

(ii) $F(x)=0$ if and only if $x=0, \pm k$ and $F(x) \rightarrow \infty$ monotonically as $x \rightarrow \infty$ for $x>k$.

(iii) $g(x)=-g(-x)$ for all $x>0$.

(iv) $g(x)>0$ for all $x>0$.

Indeed, the proof is a generalization of the existence theorem for the Van der Pol equation, to which (3.1a) reduces when $a=b=0$. The proof uses the Lyapunov function

$$
V(x, Y)=\int_{0}^{x} g(u) d u+\frac{1}{2} Y^{2},
$$

and establishes the existence of a unique stable periodic solution by considering the integral of $\dot{V}$ along orbits, and showing that there is a unique orbit sandwiched between outwardly and inwardly spirally trajectories in the $x-Y$ plane.

To apply this theorem to the present system, we first note that conditions (i) and (ii) are satisfied provided that $b<c^{2}$. Further, if $a=0$ then condition (iii) is satisfied, while condition (iv) is then also satisfied if $0 \leq b \leq 1$. Hence if $a=0$, $0 \leq b<\min \left(c^{2}, 1\right)$, we can conclude that there exists a unique stable periodic solution. Further, it can be shown that the existence theorem remains valid if condition (iv) is relaxed to the criterion that $g(x)>0$ for $0<x<x_{0}$ where $x_{0}$ is sufficiently large. Hence we conclude that the existence theorem above for a unique stable periodic solution can be extended to $a=0$ and $b<0$ provided that $|b|$ is sufficiently small. Next, when $a \neq 0$, both conditions (iii) and (iv) are violated, but the existence proof can then be adapted to hold when $|a|$ is sufficiently small, and either $0 \leq b<\min \left(c^{2}, 1\right)$, or $b<0$ and $|b|$ is sufficiently small, in which case there exists a unique stable periodic solution. 
3.4. Andronov-Hopf bifurcation: Whenever a critical point undergoes a transition from a stable to an unstable focus as some control parameter is varied, then generically a periodic solution is born through an Andronov-Hopf bifurcation. If the bifurcation is supercritical (subcritical) then the periodic solution is stable (unstable), at least in the vicinity of the bifurcation point. From our analysis in Section 2 we see that if $x_{0}$ is the critical point, then Andronov-Hopf bifurcation can only occur when $b=c^{2}\left(1-x_{0}^{2}\right)$ with $b\left(1-x_{0}^{2}\right)<1$ and $\left\{c\left(1-x_{0}^{2}\right)+b / c\right\}^{2}<4$ (see (2.7) and Figure 3 ). In particular, it follows that this can only occur if $b^{2}<c^{2}$. The periodic solution can be constructed for small amplitudes near the bifurcation point. This requires consideration of the nonlinear terms in (1.3), and the most convenient approach here is normal form analysis (for example, Grimshaw [3, pp 261-268]). We proceed to outline the application of this technique to (1.3).

First we let

$$
\begin{aligned}
\mu & =c\left(1-x_{0}^{2}\right)-\frac{b}{c}, \\
\omega^{2} & =1-b\left(1-x_{0}^{2}\right) .
\end{aligned}
$$

Here $\mu$ is the control parameter, where $\mu=0$ is the bifurcation point, while $\omega$ (at $\mu=0$ ) will be a measure of the frequency of the periodic solution generated by the bifurcation. Note that $\omega^{2}=1-b^{2} / c^{2}-\mu b / c$, and that $\omega^{2}(\mu=0)<1$. Next we let

$$
x=x_{0}+X, \quad y=y_{0}+Y,
$$

where $\left(x_{0}, y_{0}\right)$ is the critical point. Substitution into (3.5) then gives

$$
\left[\begin{array}{c}
\dot{X} \\
\dot{Y}
\end{array}\right]=A\left[\begin{array}{l}
X \\
Y
\end{array}\right]+\left[\begin{array}{c}
M \\
0
\end{array}\right]
$$

where

$$
A=\left[\begin{array}{cc}
c\left(1-x_{0}^{2}\right) & c \\
-1 / c & -b / c
\end{array}\right]
$$

and

$$
M=c\left(-x_{0} X^{2}-\frac{1}{3} X^{3}\right) .
$$

Thus $(X, Y)=(0,0)$ is now the critical point, and we recall that $x_{0}$ is defined as that solution of (2.2) corresponding to a focus, which is stable (unstable) for $\mu<0(>0$ ). The eigenvalues of the matrix $A$ are $\lambda_{1,2}(\mu)$ where

$$
\lambda_{1,2}(\mu)=\alpha(\mu) \pm i \beta(\mu)=\frac{1}{2} \mu \pm i\left(\omega^{2}-\frac{1}{4} \mu^{2}\right)^{1 / 2} .
$$


Thus $(X, Y)=(0,0)$ is a stable (unstable) focus for $\mu<0(>0)$ and $\omega^{2}>\frac{1}{4} \mu^{2}$. An Andronov-Hopf bifurcation occurs at $\mu=0$ where $\omega^{2}=\omega_{0}^{2}=1-b^{2} / c^{2}$. To construct the periodic solution emerging from this bifurcation we must consider the nonlinear term $M$ in (3.6a). This is achieved using normal form analysis.

The next step is to transform co-ordinates so that the matrix $A$ is in canonical form. Thus we put

$$
\left[\begin{array}{l}
X \\
Y
\end{array}\right]=S\left[\begin{array}{l}
\hat{X} \\
\hat{Y}
\end{array}\right]
$$

where

$$
S=\left[\begin{array}{cc}
c & 0 \\
-\alpha-b / c & \beta
\end{array}\right]
$$

Substitution into (3.6a) then gives the canonical form

$$
\left[\begin{array}{c}
\dot{\hat{X}} \\
\dot{\hat{Y}}
\end{array}\right]=\left[\begin{array}{cc}
\alpha(\mu) & \beta(\mu) \\
-\beta(\mu) & \alpha(\mu)
\end{array}\right]\left[\begin{array}{c}
\hat{X} \\
\hat{Y}
\end{array}\right]+c^{2}\left(-x_{0} \hat{X}^{2}-\frac{1}{3} c \hat{X}^{3}\right)\left[\begin{array}{c}
1 \\
(b+\alpha c) / \beta c
\end{array}\right] .
$$

Then we introduce the complex variable

$$
z=\hat{X}+i \hat{Y}
$$

so that (3.9) can be reduced to

$$
\dot{z}=(\alpha(\mu)-i \beta(\mu)) z+N(z, \bar{z} ; \mu),
$$

where

$$
N(z, \bar{z} ; \mu)=-c^{2}\left(1+\frac{i(b+\alpha c)}{\beta c}\right)\left(x_{0} \hat{X}^{2}+\frac{1}{3} c \hat{X}^{3}\right)
$$

and

$$
\hat{X}=\frac{1}{2}(z+\bar{z})
$$

Equation (3.11a) is now in a form suitable for normal form analysis as described by Grimshaw [3, p. 262]. Omitting details it can be shown that there exists a near-identity transformation $z \leftrightarrow \zeta$ such that (3.11a) is transformed into

$$
\dot{\zeta}=(\alpha(\mu)-i \beta(\mu)) \zeta+\left(\gamma(\mu)+i \delta(\mu)|\zeta|^{2} \zeta+\mathrm{O}\left(|\zeta|^{5}\right)\right)
$$


The details of this near-identity transformation need not concern us. The main issue is the calculation of the coefficients $\gamma(\mu)$ and $\delta(\mu)$. Only $\gamma(0)$ need concern us, and using the method described in Grimshaw [3,pp 267-268] we find that

$$
\gamma(0)=-\frac{c^{3}}{8 \omega_{0}^{2}}\left(1-2 b+\frac{b^{2}}{c^{2}}\right)
$$

where $\omega_{0}^{2}=\omega^{2}(\mu=0)=1-b^{2} / c^{2}$.

Equation (3.12) is readily solved using polar co-ordinates. We let $\zeta=R \exp (i \phi)$, so that

$$
\begin{aligned}
& \dot{R}=\alpha(\mu) R+\gamma(\mu) R^{3}+\mathrm{O}\left(R^{5}\right), \\
& \dot{\phi}=-\beta(\mu)+\delta(\mu) R^{2}+\mathrm{O}\left(R^{4}\right) .
\end{aligned}
$$

As well as the trivial solution $R=0$ which corresponds to the critical point $\left(x_{0}, y_{0}\right)$, equation (3.14) has a solution in which $R$ is a constant and then $\phi$ is a linear function of $t$. This corresponds to a periodic solution of $(1.3 \mathrm{a}, \mathrm{b})$, which bifurcates from $\mu=0$ and $R=0$. Near the bifurcation point this is given by

$$
R^{2} \approx-\frac{\mu}{2 \gamma(0)}
$$

Further, this solution is supercritical (subcritical) and stable (unstable) when $\gamma(0)<$ $0(>0)$. Also, from (3.14b) we see that the periodic solution has frequency $\omega_{0}+\mathrm{O}\left(R^{2}\right)$ as expected.

Consider first the case when $a=0$. If $c \leq 1$, then the bifurcation must occur from the critical point $x_{0}=0$, and occurs at $b=c^{2}$. From (3.13) it follows that $\gamma(0)=-c^{3} / 8$, and hence the bifurcation is always supercritical and generates a stable periodic solution as $b$ decreases through $c^{2}$. If $c>1$, then the bifurcation can only occur for the critical points $x_{0}= \pm\{3(1-1 / b)\}^{1 / 2}$, for some value of $b$ such that $1<b=-c^{2}+c\left(c^{2}+3\right)^{1 / 2}<3 / 2$. In this case $\gamma(0)=c^{3} / 4$ and hence the bifurcation is subcritical, and generates an unstable periodic solution from each $x_{0}$ as $b$ increases through $-c^{2}+c\left(c^{2}+3\right)^{1 / 2}$.

Next, in the more general case, when $a \neq 0$ it follows from (3.13) that any Andronov-Hopf bifurcation is always supercritical when $b \leq 0$, regardless of the values of the parameters $a$ or $c$. From Figure 3 we see that then the bifurcation occurs only for the interior critical point, and a stable periodic solution emerges. Similarly, if $b>0$ and $c \leq 1$ then it again follows from (3.13) that the bifurcation is always supercritical. From Figure 3 we see that in this case there is a single critical point. For $c>1$ and $b>0$ the situation is more complex as $\gamma(0)$ (3.13) can take both signs as $b$ varies with $c$ fixed. Indeed $\gamma(0)>0$ for $c^{2}-c\left(c^{2}-1\right)^{1 / 2}<b<c^{2}+c\left(c^{2}-1\right)^{1 / 2}$ and $\gamma(0)<0$ otherwise. But since the bifurcation can only occur for $b<c$, we infer 
that the bifurcation is subcritical for $c^{2}-c\left(c^{2}-1\right)^{1 / 2}<b<c$ leading to an unstable periodic solution, and is supercritical for $b<c^{2}-c\left(c^{2}-1\right)^{1 / 2}$, leading to a stable periodic solution. Note that here the critical point from which the bifurcation occurs is one of the outermost critical points. Also since $c^{2}-c\left(c^{2}-1\right)^{1 / 2}>1 / 2$ for all $c$, it follows that if $b<1 / 2$ the bifurcation is always supercritical.

\section{Numerical Results}

In this section we describe our results obtained from numerical integration of (1.3a-b). We use a fourth order Runge Kutta method of integration where the stepsize (usually 0.01 ) is reduced to 0.001 to ascertain the system behaviour in parameter regions where the critical points change stability or emerge, or where the behaviour of the system undergoes a transition. In general, we find that there is a unique stable periodic solution on some interval of $b$ such that $-c<b<\min \left(c, c^{2}\right)$ for appropriate fixed values of the parameters $a$ and $c$. For $c$ sufficiently close to zero, or $|a|$ sufficiently large, with the other parameters held fixed, no periodic solutions were found. But for $c>1, b>0$ and certain parameter combinations, two unstable periodic solutions were evident. These existed only for a small interval as the parameter $b$ was varied and either occurred within the stable orbit or in isolation. The periodic solutions found numerically are described in detail below, together with a qualitative discussion of the bifurcations involved and a comparison with our theoretical predictions.

For $b<0$, a supercritical Andronov-Hopf bifurcation was found when a stable focus $\left(x_{0}\right)$ lost stability as $b$ is decreased through the boundary $b=c^{2}\left(1-x_{0}^{2}\right)$ (see Figures 3(i) and (ii)). The stable periodic solution which emerged enclosed the unstable focus (which may turn into an unstable node as $b$ is decreased), but the other two critical points, both saddle points, lie outside the periodic solution (see Figure 5(i)). As $b$ decreases, the stable periodic solution grew and the saddle points approached the orbit boundary. One, or both, of the saddle points then collide with the periodic orbit, which abruptly disappears (see Figure 5(ii)). This is an example of a blue-sky catastrophe, and can be predicted using index laws (see 3.2), although we are unable to obtain an exact analytical expression of the parameters where this occurs. However, the birth of the periodic orbit as a supercritical Andronov-Hopf bifurcation is predicted in 3.4 .

For $b \geq 0, c \leq 1$ and $|a| \leq 1$, the same birth and growth of a periodic solution was found as $b$ is decreased through $b=c^{2}\left(1-x_{0}^{2}\right)$, and is again a supercritical Andronov-Hopf bifurction as predicted in 3.4. The cycle persisted for $b<0$ before disappearing through a blue-sky catastrophe for some $b$ in the range $-c<b<0$. For $b \geq 0, c \leq 1$ and $|a|>1$ no critical point can change stability and hence no stable periodic solutions can emerge through an Andronov-Hopf bifurcation. 
For $0<b<c^{2}-c \sqrt{c^{2}-1}, c \geq 1$ and $|a| \leq 1$, there is again the same situation described in the preceeding paragraph for the case $b \geq 0, c \leq 1$ and $|a| \leq 1$. That is, a supercritical Andronov-Hopf bifurcation gives rise to a stable periodic solution, which grows as $b$ is decreased until for some $b$ in the range $-c<b<0$ it disappears through a blue-sky catastrophe.

For $b>c^{2}-c \sqrt{c^{2}-1}, c \geq 1$ and $|a| \leq 1$, the situation is more complex. A stable periodic solution exists for $b<c^{2}\left(1-x_{0}^{2}\right)$, and as $b$ decreases, is annihilated when one (or both) saddle points collide with it for some $b,-c<b<0$. However, if $b$ is increased through $b=c^{2}\left(1-x_{0}^{2}\right)$ an unstable periodic solution (two distinct unstable periodic solutions if $a=0$ ) emerges within the stable cycle, as an unstable focus gains stability. Four distinct scenarios were evident for different values of the parameter $a$, as $b$ is varied. These are described below and illustrated in Figure 8, where the dotted (solid) lines represent the unstable (stable) periodic solutions.

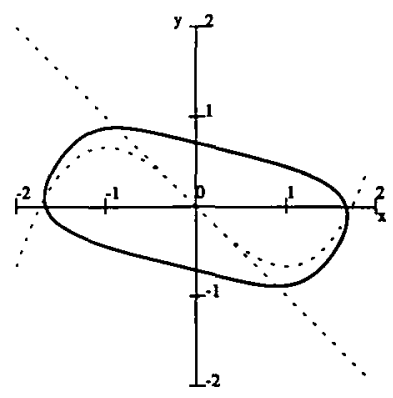

Parmeters a, b.c :- 0.01 .00020

(i)

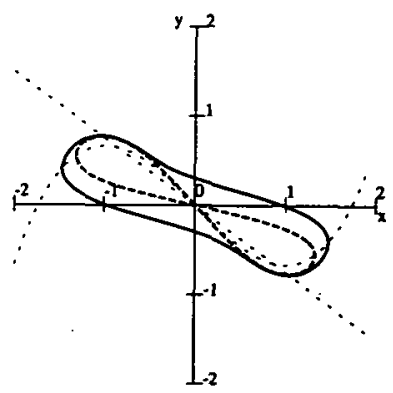

Perameters a, b, $c:-0.013372 .0$

(iii)

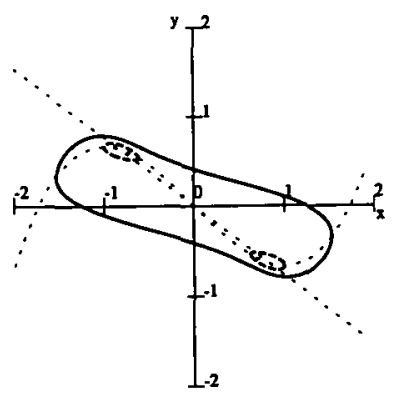

Paranders a, b. :- 0.0130020

(ii)

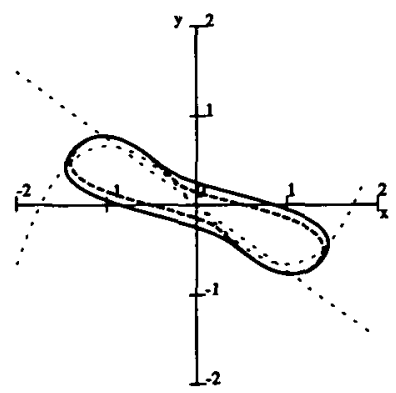

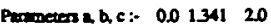

(iv)

- sable periodic solutions

FIGURE 8 (CASE I) Phase plane for the BVP system 


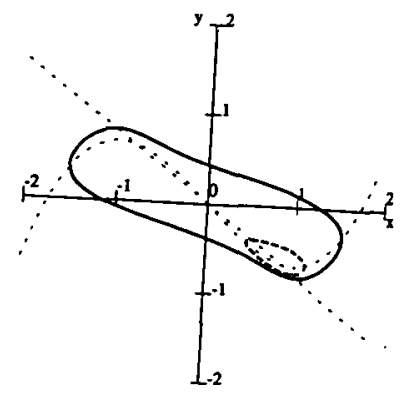

Parameters a, b, $c$ :- 0.011 .3012 .0

(i)

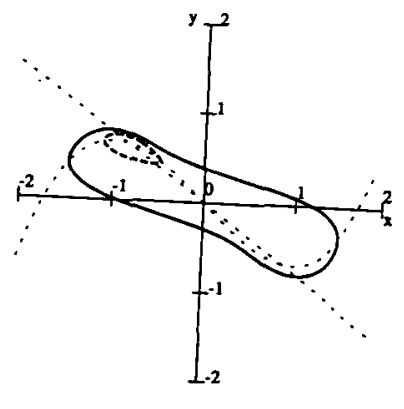

Peraneters a, b. c:- $0.01 \quad 1.3252 .0$

(iii)

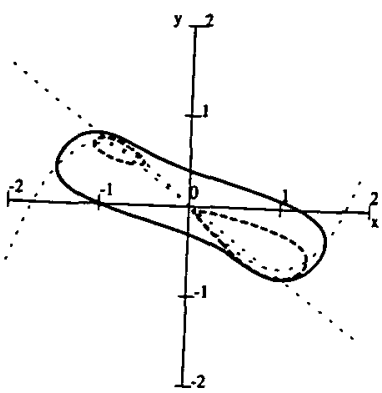

Parmeters a b. c:- $0.01 \quad 1.322 \quad 2.0$

(ii)

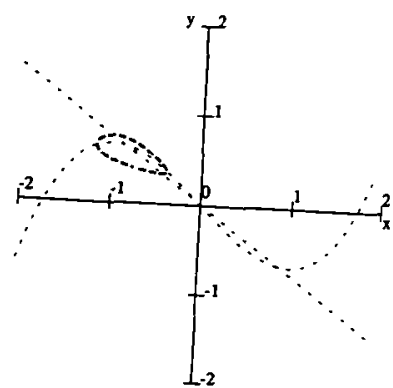

Parameters a b.c:- $0.01 \quad 1.3332 .0$

(iv)

-..

FIGURE 8 (CASE II) Phase plane for the BVP system

First, for $a=0$, the system is symmetrical (see Figure 8, Case I). As $b$ is increased through $b=c^{2}\left(1-x_{0}^{2}\right)$, two distinct unstable orbits emerge simultaneously, each enclosing a single stable focus, with a saddle point at the origin lying outside both orbits, and between them. The birth of these unstable cycles is a subcritical AndronovHopf bifurcation as predicted in 3.4. Both these unstable periodic cycles and the saddle point are enclosed within a stable periodic solution. As $b$ is increased further, the unstable periodic solutions grow, collide with the saddle point, and then merge to form a single unstable periodic solution, enclosing all three critical points and enclosed within the stable cycle. Further increase of $b$ then leads to coalescence of these cycles and annihilation of both. This is an example of a cyclic-fold bifurcation or a saddle-node bifurcation in a Poincaré map.

Next, we suppose $a \neq 0$ but $|a| \ll 1$ (see Figure 8 , Case II). Now as $b$ increases through $b=c^{2}\left(1-x_{0}^{2}\right)$ a single unstable periodic solution emerges enclosing a 


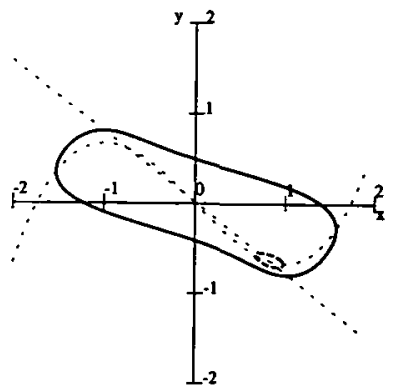

Paramevers a. b. c: -0.021 .27020$

(i)

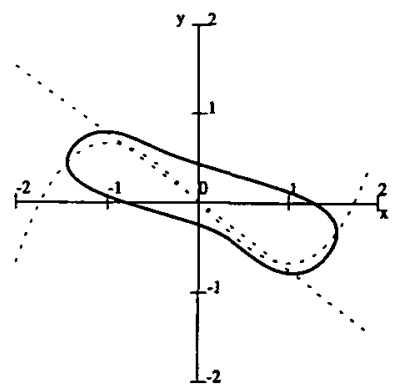

Parameters a, b. c : 0.021 .3152 .0

(iii)

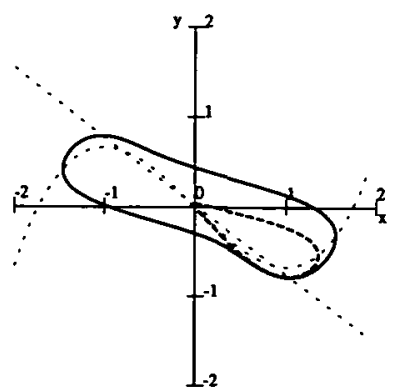

Pandeter in b, $c:-0.02131020$

(ii)

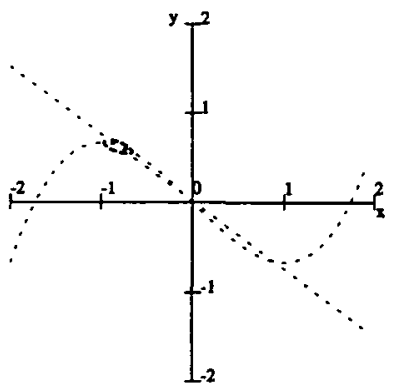

Pandineter a b, c:- 0.061 .3252 .0

(iv)

-.-- unstable periodic solutions

FIGURE 8 (CASE III) Phase plane for the BVP system

stable focus, which is the only critical point. Both are enclosed inside a stable cycle. As $b$ continues to increase, a saddle point and an unstable node or focus emerge simultaneously (see Figure 3 ). The latter critical point gains stability as $b$ increases, and this leads to the birth of a second unstable periodic solution. Both these unstable periodic solutions are born through subcritical Andronov-Hopf bifurcations as predicted in 3.4. At this stage there is a stable cycle, enclosing two unstable cycles, with a saddle point between them. As $b$ continues to increase, the unstable cycles expand until one collides with the saddle point and is annihilated by a blue-sky catastrophe. Next, as $b$ increases the stable cycle is annihilated when it collides with the stable manifold emerging from the saddle point. Finally, as b continues to increase, the unstable cycle disappears when it collides with the saddle point.

Now we let $|a|$ increase a little, but still have $|a| \ll 1$ (see Figure 8, Case III). As $b$ increases through $b=c^{2}\left(1-x_{0}^{2}\right)$, a single unstable cycle emerges enclosing 


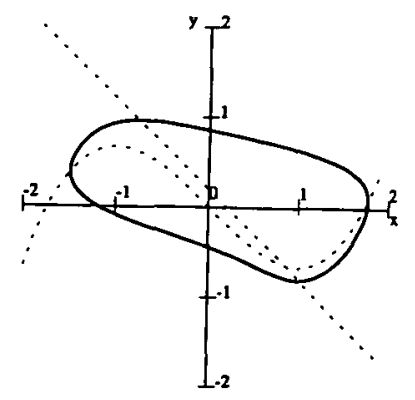

Parmeters a, b.c: : 0.21 .0002 .0

(i)

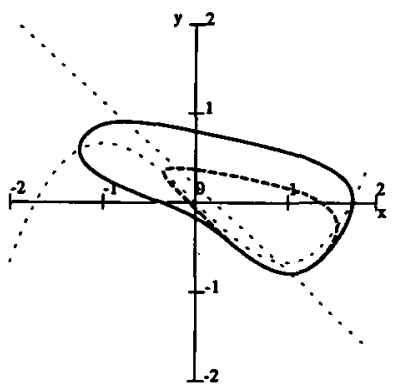

Permeteri a, b. : : 0.201 .06020

(iii)

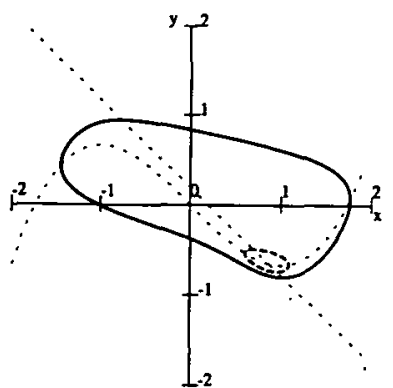

Permeters a, b, c:- 0.201 .03020

(ii)

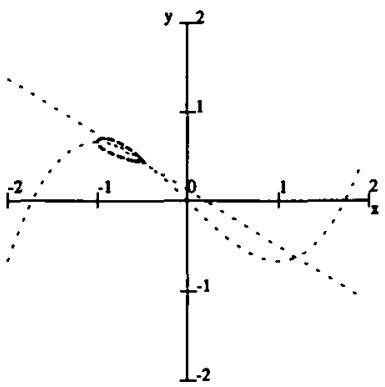

Punewers a, b:- 0.20159520

(iv)

\section{- - - wheric pedic colutions}

Figure 8 (CASE IV) Phase plane for the BVP system

a stable focus, and both are enclosed by a stable cycle. At this stage this is similar to Case II, and as $b$ continues to increase an unstable node and saddle point emerge. But in contrast to Case II this unstable node, or focus, did not change stability as $b$ increases before the unstable cycle collided with the saddle point, and was annihilated through a blue-sky catastrophe. Further increase in $b$ then leads to the annihilation of the stable cycle when it collides with the stable manifold from the saddle point, and then there are no periodic solutions. But as $b$ continues to increase, another unstable cycle is bom through a subcritical Andronov-Hopf bifurcation (see 3.4), and it in turn is annihilated when it collides with the saddle point.

The fourth case occurs when $|a|$ is larger but still $|a|<1$ (see Figure 8, Case IV). As in Cases II and III a stable periodic solution exists enclosing an unstable node or focus. As $b$ increases through $b=c^{2}\left(1-x_{0}^{2}\right)$ this changes stability, and an unstable periodic solution is born through a subcritical Andronov-Hopf bifurcation 
as predicted in 3.4. But in contrast to Cases II and III, as $b$ continues to increase, no further critical points emerge and instead the unstable periodic solution collides with the stable cycle, with both being annihilated through a cyclic-fold bifurcation (or a saddle-node bifurcation in the Poincaré map). Further increase in $b$ then leads to the emergence of a saddle point and a stable node. If $b>c^{2}\left(1-x_{0}^{2}\right)$ for this node/focus then it remains stable as $b$ increases, and there are no more periodic solutions (see Figure 3). But if $b<c^{2}\left(1-x_{0}^{2}\right)$ for this node/focus when it is born, then as $b$ increases through $b=c^{2}\left(1-x_{0}^{2}\right)$, an unstable cycle emerges through an Andronov-Hopf subcritical bifurcation as predicted in 3.4. For further increase in $b$ it is annihilated when it collides with the saddle point.

Thus the Andronov-Hopf bifurcation theory described in 3.4 was successful in predicting all cases of the emergence of periodic solutions, whether stable or unstable. In general, the theory of Section 3 gives a very good indication of when to expect periodic solutions to occur. In Figure 9 we compare the theoretical predictions for the existence of periodic solutions in the $b-a$ parameter space with the theoretical predictions shown in Figure 7. We see there is good agreement. The dotted lines indicate where stable periodic solutions were found for the indicated values of $a$. The solid lines (short in length) appearing in the narrow unshaded tails to the right of the figure in $b>0$ are unstable periodic solutions appearing in isolation. The short solid lines appended to the dotted lines in $b \geq 0$ indicate where stable and unstable periodic solutions appeared simultaneously.

\section{Summary}

In this paper we have reconsidered the Bonhoeffer van der Pol (BVP) system (1.1a, b) introduced by Fitzhugh $[1,2]$ as a model of a physiological system, obtained in the first instance as a two-dimensional projection of the four-dimensional projection of the well-known Hodgkin-Huxley system. We have extended Fitzhugh's analysis from a limited portion of the parameter space to the full parameter space, for the case when the applied impulse is a constant so that the sysem is planar and autonomous. In a part of the parameter space which includes Fitzhugh's results we have found that the system exhibits a stable periodic solution, as well as sometimes unstable periodic solutions occuring simultaneously for a few cases of the same parameter values. Typically this situation occurs when there is just a single critical point. But in the full parameter space, as well as the case of just a single critical point with the possibility of a unique stable periodic orbit, the system can exhibit three critical points with the possibility of up to three periodic solutions existing simultaneously. Our results are obtained using both theoretical analyses and numerical solutions, and we find that together these provide a consistent picture of the system behaviour. In particular, 


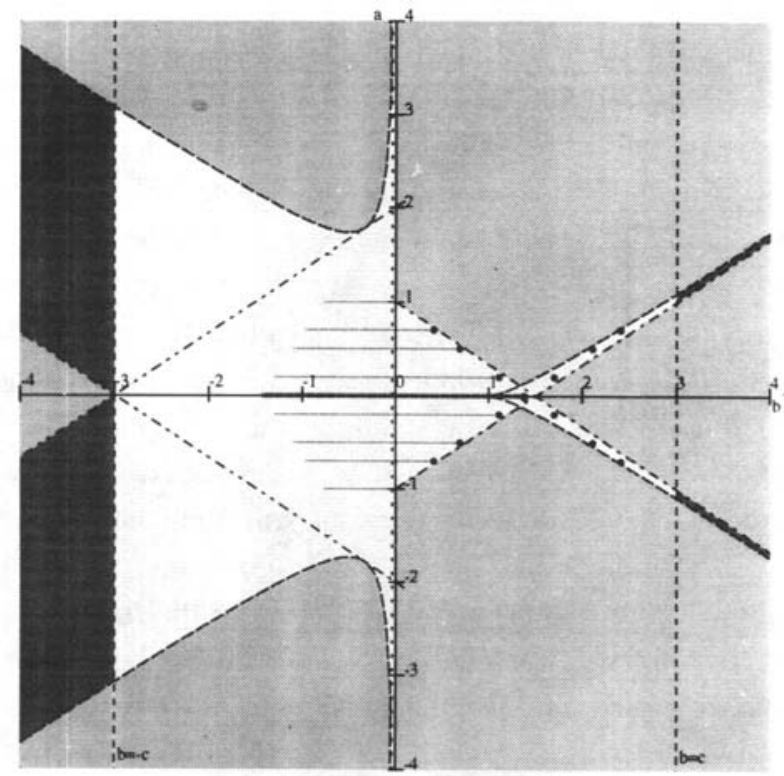

Represented in the b-a parameter space with $c=3$

Stable periodic solutions Unstable periodic solutions occuring simultaneously with a stable periodic solution. when the solution regions ane adjoined $\mathrm{a}=2 \mathrm{~b} / 3-1$

$a=2 / 3\left((b-1)^{3} / b^{\mu}\right.$.

No periodic solutions or possible Hopr bifurcalion

Further areas where Hopf bifurcation cannox occur for a particular fixed $\mathrm{c}(\mathrm{c}=3$.)

FIGURE 9. Numerically found periodic solutions

our theoretical predictions successfully describe the emergence and annihilation of periodic solutions.

It was not the primary purpose of this paper to compare our results with a specific physiological system, such as the Hodgkin-Huxley system, or to relate our results to any particular physiological application. However, it should be noted that both stable and unstable periodic solutions have been found in the Hodgkin-Huxley system (Rinzel and Miller, [9]). Indeed for the Hodgkin-Huxley system it has been shown that a stable periodic solution exists within some parameter interval of the applied impulse strength, and that unstable periodic solutions can co-exist within a subinterval of this parameter range. The periodic solutions emerged through an Andronov-Hopf bifurcation (Rinzel, [8]). Further, the emergence of an unstable periodic solution through an Andronov-Hopf bifurcation which grows in amplitude before colliding with the stable periodic solution enclosing it, followed by the annihilation of both 
in a cyclic-fold bifurcation (see, Figures $8 \mathrm{I}$ and $8 \mathrm{IV}$ ), is one of the features of the Hodgkin-Huxley system (Guttman, Lewis and Rinzel, [4]), and has been found here also for the BVP system. Thus there are several qualitiative similarities of the BVP system with the Hodgkin-Huxley system, confirming that the BVP system is a robust model of certain physiological phenomena. However, it remains to establish exact connections betwen the parameters $a, b, c$ of the BVP system and actual physiological variables.

\section{References}

[1] R. Fitzhugh, "Threshholds and plateaus in the Hodgkin-Huxley nerve equations", J. Gen. Physiol. 45 (1960) 867-896.

[2] R. Fitzhugh, "Impulses and physiological states in models of nerve membrane", Biophys. J. 1 (1961) 445-466.

[3] R. Grimshaw, Nonlinear ordinary differential equations (Blackwell, Oxford, 1990).

[4] R. Guttman, S. Lewis and J. R. Rinzel, "Control of repetitive firing in squid axon membrane as a model for a neuronoscillator", J. Physiol. 305 (1980) 377-395.

[5] A. L. Hodgkin and A. F. A. Huxley, "A quantitative description of membrane current and its application to conduction and excitation in nerve", J. Physiol. 117 (1952) 500-544.

[6] D. W. Jordan and P. Smith, Nonlinear ordinary differential equations, second ed. (Oxford University Press, Oxford, 1987).

[7] P. E. Kloeden, E. Platen and H. Shurz, Numerical solution of stochastic differential equations through computer experiments (Springer, Berlin, 1994).

[8] J. Rinzel, "Excitation dynamics: insights from simplified membrane models", Theoretical Trends on Neuroscience 44 (1985) 2945-2946.

[9] J. Rinzel and R. N. Miller, "Numerical calculation of stable and unstable periodic solutions in the Hodgkin-Huxley equations", Math. Biosciences 49 (1980) 27-59.

[10] S. Wiggins, Introduction to applied nonlinear dynamical systems and chaos (Springer, New York, 1990). 